\title{
A Critique of the Random Intercept Cross-Lagged Panel Model
}

\author{
Oliver Lüdtke ${ }^{1,2}$ \& Alexander Robitzsch ${ }^{1,2}$ \\ ${ }^{1}$ IPN - Leibniz Institute for Science and Mathematics Education, Kiel, Germany \\ ${ }^{2}$ Centre for International Student Assessment (ZIB), Kiel, Germany
}

\begin{abstract}
The random intercept cross-lagged panel model (RI-CLPM) is an extension of the traditional cross-lagged panel model (CLPM) that allows controlling for stable trait factors when estimating cross-lagged effects. It has been argued that the RI-CLPM more appropriately accounts for trait-like, timeinvariant stability of many psychological constructs and that it should be preferred over the CLPM when at least three waves of measurement are available. The basic idea of the RI-CLPM is to decompose longitudinal associations between two constructs into stable between-person associations and temporal within-person dynamics. The present article critically examines the RI-CLPM from a causal inference perspective. Using formal analysis and simulated data, we show that the RI-CLPM has limited potential to control for unobserved stable confounder variables when estimating crosslagged effects. The CLPM with additional lag-2 effects sufficiently controls for delayed effects, as long as all relevant covariates are measured. Furthermore, we clarify that, in general, the RI-CLPM targets a different causal estimand than the CLPM. Whereas the cross-lagged effect in the CLPM targets the effect of increasing the exposure by one unit, the within-person cross-lagged effect in the RI-CLPM provides an estimate of the effect of increasing the exposure by one unit around the person mean. We argue that this within-person causal effect is typically less relevant for testing causal hypotheses with longitudinal data because it only captures temporary fluctuations around the individual person means and ignores the potential effects of causes that explain differences between persons.
\end{abstract}

Keywords: cross-lagged panel model, random intercept cross-lagged panel model, cross-lagged effect, causal inference, longitudinal data, reciprocal effects

\section{Introduction}

In many areas of psychological research, longitudinal cross-lagged panel designs are used to investigate whether changes in one construct $X$ are related to changes in another 
construct $Y$ (Little, 2013; Marsh, Trautwein, Lüdtke, Köller, \& Baumert, 2005; Orth, Clark, Donnellan, \& Robins, 2021). In the basic setting of two measurement waves, the key idea of the cross-lagged panel model (CLPM) is that the effect of a predictor at T1 on an outcome at T2 (i.e., cross-lagged effect) is estimated, controlling for the outcome at T1. Thus, the CLPM is based on a conditioning approach in which the posttest $\left(Y_{2}\right)$ is conditioned on the pretest $\left(Y_{1}\right)$ by regressing the posttest on the pretest and a potential treatment variable ( $X_{1}$; Maxwell \& Delaney, 2004; Newsom, 2015; Plewis, 1985).

However, in a very influential paper (1103 citations listed on Google Scholar as of August 12, 2021), Hamaker, Kuiper, and Grasman (2015) criticized that the CLPM does not appropriately account for the trait-like, time-invariant stability of many psychological constructs and results in distorted estimates of cross-lagged effects. Hamaker et al. (2015) proposed the random intercept cross-lagged panel model (RI-CLPM) as an extension of the traditional CLPM that allows controlling for stable trait factors when at least three measurement waves are available (Usami, Murayama, \& Hamaker, 2019). More specifically, the RI-CLPM decomposes longitudinal associations between two constructs into stable betweenperson associations (i.e., the correlation between time-invariant between-person parts) and temporal within-person dynamics (i.e., within-person effects for deviations from betweenperson parts). This decomposition allows estimating within-person cross-lagged effects that are adjusted for the effects of stable trait factors. The RI-CLPM has received considerable attention in the methodological literature. Many scholars argue that the RI-CLPM should be preferred over the CLPM for estimating cross-lagged effects, particularly in the presence of stable trait factors (e.g., Berry \& Willoughby, 2017; Curran \& Hancock, 2021; Grimm, Helm, Rodgers, \& O'Rourke, 2021; Mulder \& Hamaker, 2021; Mund \& Nestler, 2019; Usami, 2021; Zyphur et al., 2020). Furthermore, empirical comparisons of the CLPM and the RI-CLPM have shown that the decision between the CLPM and RI-CLPM is crucial because the two approaches can yield results that substantially differ concerning the magnitude, sign, and statistical significance of the estimated cross-lagged effect (e.g., Bailey, Oh, Farkas, Morgan, \& Hillemeier, 2020; Ehm, Hasselhorn, \& Schmiedek, 2019; Littlefield et al., 2021; Oh, Greenberg, \& Willoughby, 2020; Orth et al., 2021; Ruzek \& Schenke, 2019; Zhou et al., 2020).

One frequently made argument in favor of the RI-CLPM is that it offers the option to control for unobserved confounding variables that are stable across time (Usami, Murayama, \& Hamaker, 2019; see also Bailey et al., 2020). Given that one of the main challenges in estimating causal effects with non-experimental data is to control for all relevant covariates (Reichardt, 2019), this seems to be a significant advantage of the RI-CLPM. In the present article, we compare the CLPM and the RI-CLPM from a causal perspective and discuss under which assumptions the two approaches provide unbiased estimates of a cross-lagged effect. More specifically, we apply a structural modeling perspective to a cross-lagged panel design with three waves of measurement (Pearl, Glymour, \& Jewell, 2016) and define the causal estimand (i.e., the cross-lagged effect) using potential outcome notation (Imbens \& Rubin, 2015). For this set-up, we show that very strong assumptions about the effects of the confounder variable need to be met for the RI-CLPM to provide unbiased effects of the cross-lagged effect and that, therefore, the RI-CLPM has only limited potential to control for an unobserved time-invariant confounder. We also show that it is often advisable to include lag-2 effects (i.e., effects of variables across two units of time) in addition to lag-1 effects 
in the CLPM in order to control for delayed effects of $X$ and $Y$ (VanderWeele, Mathur, \& Chen, 2020). Finally, we clarify that, in general, the RI-CLPM estimates a different causal estimand (i.e., the target of inference) than the CLPM. Whereas the cross-lagged effect in the CLPM targets the effect of increasing the exposure by one unit, the within-person crosslagged effect in the RI-CLPM provides an estimate of the effect of increasing the exposure by one unit around the person mean. We argue that this within-person causal effect typically is of less interest for testing causal hypotheses with longitudinal data because it only captures temporary fluctuations around the individual person means and ignores long-term effects that persist over time (Asendorpf, 2021; Orth et al., 2021). Overall, the goal of this paper is to provide a more balanced discussion of two main approaches (CLPM and RI-CLPM) for analyzing cross-lagged panel designs, and we would like to emphasize that - despite recent methodological recommendations - there are still good reasons to use the traditional CLPM when estimating cross-lagged effects.

Before we start, we would like to point out that at a more descriptive level, the traditional CLPM has been criticized by methodologists and developmental psychologists with the argument that it provides an uninterpretable blend of within-person effects and between-person effects (e.g., Berry \& Willoughby, 2017; Hamaker et al., 2015). In the present article, we focus on the question under which conditions cross-lagged effects in the RI-CLPM can be given a causal interpretation. The question of whether the decomposition into within-person and between-person effects (in contrast to the undecomposed effects in the CLPM) provides a more appropriate description of longitudinal processes will be discussed in the final section.

\section{Modeling Cross-Lagged Effects with the CLPM and RI-CLPM}

In the following, we assume a longitudinal panel design with three waves in which two variables are repeatedly measured at equally spaced intervals over time. Let us assume $Y_{i t}$ and $X_{i t}$ (e.g., self-esteem and depression) were assessed for persons $i(i=1, \ldots, N)$ across time $t(t=1,2,3)$. In the typical application, researchers are interested in whether there exist effects from one variable on another variable over time (i.e., effects of self-esteem on subsequent depression). We start with the traditional CLPM.

\subsection{Cross-Lagged Panel Model with Lag-1 Effects (CL1PM)}

The traditional CLPM model (Finkel, 1995; Kessler \& Greenberg, 1981; Little, 2013) is given by (see Figure 1):

$$
\begin{aligned}
X_{i t} & =\beta_{0 X_{t}}+\beta_{X_{t} X_{t-1}} X_{i, t-1}+\beta_{X_{t} Y_{t-1}} Y_{i, t-1}+d_{X i t} \\
Y_{i t} & =\beta_{0 Y_{t}}+\beta_{Y_{t} Y_{t-1}} Y_{i, t-1}+\beta_{Y_{t} X_{t-1}} X_{i, t-1}+d_{Y i t}
\end{aligned}
$$

where $\beta_{0 X_{t}}$ and $\beta_{0 Y_{t}}$ are the regression intercepts, $\beta_{X_{t} X_{t-1}}$ and $\beta_{Y_{t} Y_{t-1}}$ are the autoregressive parameters that indicate the stability across time (e.g., the extent to which $X_{i t}$ depends on the previous time $X_{i, t-1}$; first-order autoregression), and $\beta_{X_{t} Y_{t-1}}$ and $\beta_{Y_{t} X_{t-1}}$ are the crosslagged parameters. The residuals $d_{X i t}$ and $d_{Y i t}$ denote correlated random components that are unrelated to previous time points and are normally distributed with zero means. If both cross-lagged parameters are significantly different from zero, the two variables $X_{i t}$ and $Y_{i t}$ 
are said to be reciprocally related. It should be emphasized that the CLPM includes one of the most basic and frequently used analysis strategies in psychology (Orth et al., 2021). It is based on two regression models that each estimate the effect of an exposure (e.g., $X_{i, t-1}$ ) on an outcome (e.g., $Y_{i t}$ ) measured at a later time point while controlling for the previous values of the outcome $\left(Y_{i, t-1}\right.$; see VanderWeele, Jackson, \& Li, 2016). Thus, the CLPM controls for preexisting differences in the outcome when assessing the effect of the exposure and is a special case of a more general class of conditioning methods in which an estimate of the causal effect is obtained by conditioning on the pretest and other observed covariates. Further covariates $Z_{i}$ that are assumed to be constant across the investigated time period (e.g., gender, SES) are easily included in the CLPM by regressing both $X_{i t}$ and $Y_{i t}$ on $Z_{i}$.

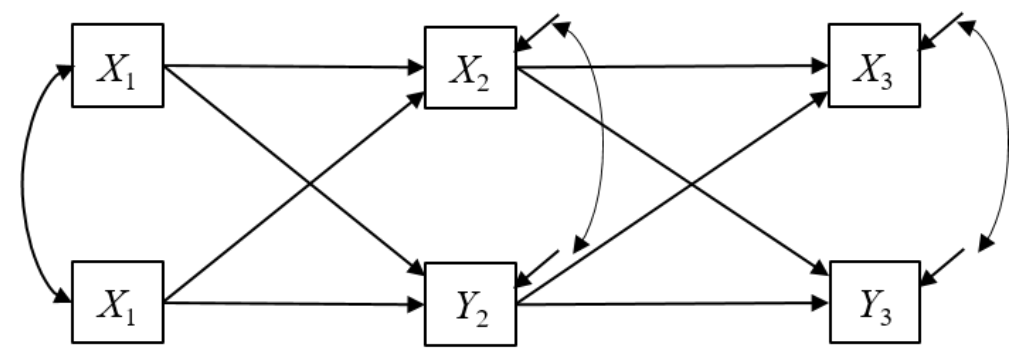

Figure 1. Path diagram of the cross-lagged panel model with lag-1 effects (CL1PM) for three measurement waves.

The CLPM can be estimated with only two waves of data. With more than two waves, the autoregressive and cross-lagged parameters are often constrained to be invariant across waves. This can be reasonable if the time intervals between the waves have a similar length (Little, 2013). As the traditional CLPM only considers the effects of the previous measurement wave (i.e., lag-1 effects), we will refer to the model in Equation 1 as CL1PM. It needs to be emphasized that previous comparisons of the CLPM and RI-CLPM (e.g., Bailey et al., 2020; Dietvorst, Hiemstra, Hillegers, \& Keijsers, 2018; Littlefield et al., 2021; Usami, Murayama, \& Hamaker, 2019) mainly focused on the CL1PM and did not consider the role of higher-order lags (e.g., lag-2 effects).

\subsection{Cross-Lagged Panel Model with Lag-2 Effects (CL2PM)}

With three or more waves, the CL1PM can be extended to a CL2PM that includes the effects of variables from two previous time points (i.e., lag-2 effects):

$$
\begin{aligned}
& X_{i t}=\beta_{0 X_{t}}+\beta_{X_{t} X_{t-1}} X_{i, t-1}+\beta_{X_{t} X_{t-2}} X_{i, t-2}+\beta_{X_{t} Y_{t-1}} Y_{i, t-1}+\beta_{X_{t} Y_{t-2}} Y_{i, t-2}+d_{X i t} \\
& Y_{i t}=\beta_{0 Y_{t}}+\beta_{Y_{t} Y_{t-1}} Y_{i, t-1}+\beta_{Y_{t} Y_{t-2}} Y_{i, t-2}+\beta_{Y_{t} X_{t-1}} X_{i, t-1}+\beta_{Y_{t} X_{t-2}} X_{i, t-2}+d_{Y i t}
\end{aligned}
$$

where $\beta_{X_{t} X_{t-2}}$ and $\beta_{Y_{t} Y_{t-2}}$ denote the lag-2 stability effects (e.g., the extent to which $X_{i t}$ depends on $X_{i, t-2}$ over and above the effect of $X_{i, t-1}$; second-order autoregression), and $\beta_{X_{t} Y_{t-2}}$ and $\beta_{Y_{t} X_{t-2}}$ are the lag-2 cross-lagged effects (see Figure 2).

The CL1PM is a special case of the CL2PM with only lag-1 effects and is thus nested within the CL2PM. There are different perspectives on the importance of including these 


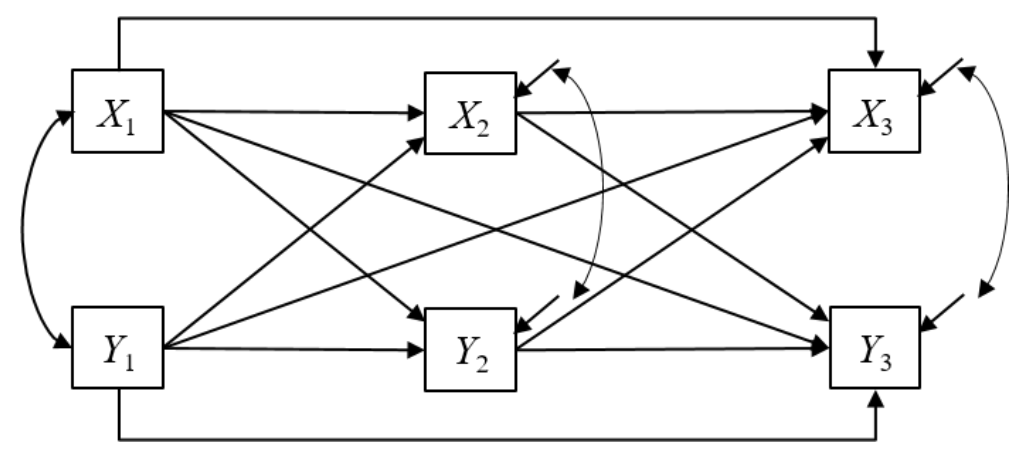

Figure 2. Path diagram of the cross-lagged panel model with lag-1 and lag-2 effects (CL2PM) for three measurement waves.

lag-2 effects in the CL2PM (Little, 2013). One view is that they consider delayed effects that are not captured by the lag-1 effects (e.g., Asendorpf, 2021; Marsh et al., 2018). However, it has been argued that it is often difficult to justify which psychological mechanism could be responsible for these delayed effects and that direct effects (i.e., lag-1 effects) are often more plausible (e.g., Ehm et al., 2019). From a causal inference perspective, the main motivation for including lag-2 effects is a more comprehensive control for confounding (VanderWeele et al., 2020). If one is interested in estimating the cross-lagged effect of $X_{i 2}$ on $Y_{i 3}$, the previous exposure $\left(X_{i 1}\right)$ and outcome measures $\left(Y_{i 1}\right.$ and $\left.Y_{i 2}\right)$ can be considered covariates that allow for stronger control of confounder variables. VanderWeele et al. (2020, p. 444) write in their detailed discussion of the benefits of controlling for prior values of the exposure (i.e., controlling for the effects of $X_{i 1}$ when the main goal is to estimate the effect of $X_{i 2}$ on $\left.Y_{i 3}\right)$ : "Control for prior exposure can also help further rule out other forms of unmeasured confounding. This is so because, if control is made for prior exposure then, for unmeasured confounder $U$ to explain away an observed exposure-outcome association, the unmeasured confounder would have to be associated with both the outcome and the baseline exposure, independent of the prior level of exposure." Potential challenges when controlling for lag-2 effects of the exposure $X$ will be addressed in the final discussion.

\subsection{Random Intercept Cross-Lagged Panel Model (RI-CLPM)}

The RI-CLPM is an extension of the traditional CL1PM with lag-1 effects. It includes additional latent variables (also labeled as random intercepts; Hamaker et al., 2015) that model the stable, time-invariant part of the observed variables (see Figure 3). More specifically, the observed $X_{i t}$ and $Y_{i t}$ are given as

$$
\begin{aligned}
X_{i t} & =\mu_{X_{t}}+I_{X i}+X_{i t}^{*} \\
Y_{i t} & =\mu_{Y_{t}}+I_{Y i}+Y_{i t}^{*}
\end{aligned},
$$

where $I_{X i}$ and $I_{Y i}$ are time-invariant stable trait factors representing the parts of $X_{i t}$ and $Y_{i t}$ that are stable across time (e.g., a completely stable component of self-esteem). The residuals $X_{i t}^{*}$ and $Y_{i t}^{*}$ are time-specific individual deviations from the time-invariant random intercepts $I_{X i}$ and $I_{Y i}$. As pointed out by Hamaker et al. (2015), the RI-CLPM adopts 
a multilevel perspective in which the individual measurements are nested within persons, and the observed measurements are decomposed into within-person parts (i.e., $X_{i t}^{*}$ and $Y_{i t}^{*}$ ), and between-person parts (i.e., $I_{X i}$ and $I_{Y i}$ ). At the between-person level, the two timeinvariant stable trait factors are allowed to be correlated (e.g., whether individuals with high self-esteem, in general, are also more depressive; denoted as $\left.\phi_{I X Y}\right)$. However, the withinperson and between-person parts are orthogonal (i.e., uncorrelated) so that the RI-CLPM separates stable between-person differences (i.e., stable trait factors) from within-person fluctuations over time (captured by the within-person parts $X_{i t}^{*}$ and $Y_{i t}^{*}$ ).

At the within-person level, the effects among the time-specific individual deviations $X_{i t}^{*}$ and $Y_{i t}^{*}$ are modeled as:

$$
\begin{aligned}
X_{i t}^{*} & =\beta_{X_{t}^{*} X_{t-1}^{*}} X_{i, t-1}^{*}+\beta_{X_{t}^{*} Y_{t-1}^{*}} Y_{i, t-1}^{*}+d_{X i t}^{*} \\
Y_{i t}^{*} & =\beta_{Y_{t}^{*} Y_{t-1}^{*}} Y_{i, t-1}^{*}+\beta_{Y_{t}^{*} X_{t-1}^{*}} X_{i, t-1}^{*}+d_{Y i t}^{*}
\end{aligned}
$$

where the coefficients $\beta_{X_{t}^{*} X_{t-1}^{*}}$ and $\beta_{Y_{t}^{*} Y_{t-1}^{*}}$ represent the autoregressive effects and the coefficients $\beta_{X_{t}^{*} Y_{t-1}^{*}}$ and $\beta_{Y_{t}^{*} X_{t-1}^{*}}$ represent the cross-lagged effects. The cross-lagged effects can now be interpreted as within-person effects. For example, $\beta_{Y_{t}^{*} X_{t-1}^{*}}$ indicates whether a temporal deviation (i.e., $X_{i, t-1}^{*}$ ) from the stable trait level (i.e., random intercept $I_{X i}$ ) of one construct affects subsequent within-person deviations (i.e., $Y_{i t}^{*}$ ) from the stable trait level (i.e., random intercept $I_{Y i}$ ) of the other construct.

To further emphasize the multilevel structure of the RI-CLPM, it is instructive to merge Equations (3) and (4) into

$$
\begin{aligned}
X_{i t} & =\mu_{X_{t}}+I_{X i}+\beta_{X_{t}^{*} X_{t-1}^{*}} X_{i, t-1}^{*}+\beta_{X_{t}^{*} Y_{t-1}^{*}} Y_{i, t-1}^{*}+d_{X i t}^{*} \\
Y_{i t} & =\mu_{Y_{t}}+I_{Y i}+\beta_{Y_{t}^{*} Y_{t-1}^{*}} Y_{i, t-1}^{*}+\beta_{Y_{t}^{*} X_{t-1}^{*}} X_{i, t-1}^{*}+d_{Y i t}^{*}
\end{aligned}
$$

From the perspective of the RI-CLPM, it can be argued (Hamaker et al., 2015) that the cross-lagged parameters of the CL1PM (i.e., $\beta_{X_{t} Y_{t-1}}$ and $\beta_{Y_{t} X_{t-1}}$; see Equation (1)) that does not include the stable trait factors (i.e., $I_{X i}$ and $I_{Y i}$ ) conflate within-person and between-person effects and provide an "uninterpretable weighted blend of between- and within-person effects" (Berry \& Willoughby, 2017, p. 1194). However, this argument is based on the assumption that the RI-CLPM is the true data-generating model. As will be shown later, an alternative view is that the delayed effects (i.e., lag-2 effects) of the CL2PM are responsible for the stable trait factors. From this perspective, the CL1PM needs to be extended to the CL2PM, but no decomposition into within-person and between-person parts is necessary to capture the effects of additional stabilizing influences.

The RI-CLPM requires that at least three waves of measurements are available to be identified. The main difference between the CL1PM and the RI-CLPM is that the RICLPM includes stable trait factors $I_{X i}$ and $I_{Y i}$. In this sense, the RI-CLPM is the more general model, and the CL1PM is nested under the RI-CLPM (i.e., variances and covariance of stable trait factors are constrained to zero). Thus, model comparisons can be used to test whether the RI-CLPM better fits the empirical data (e.g., chi-square difference tests). However, it can be debated whether the model fit is an appropriate criterion for deciding between the CL1PM and the RI-CLPM (Orth et al., 2021). 
7

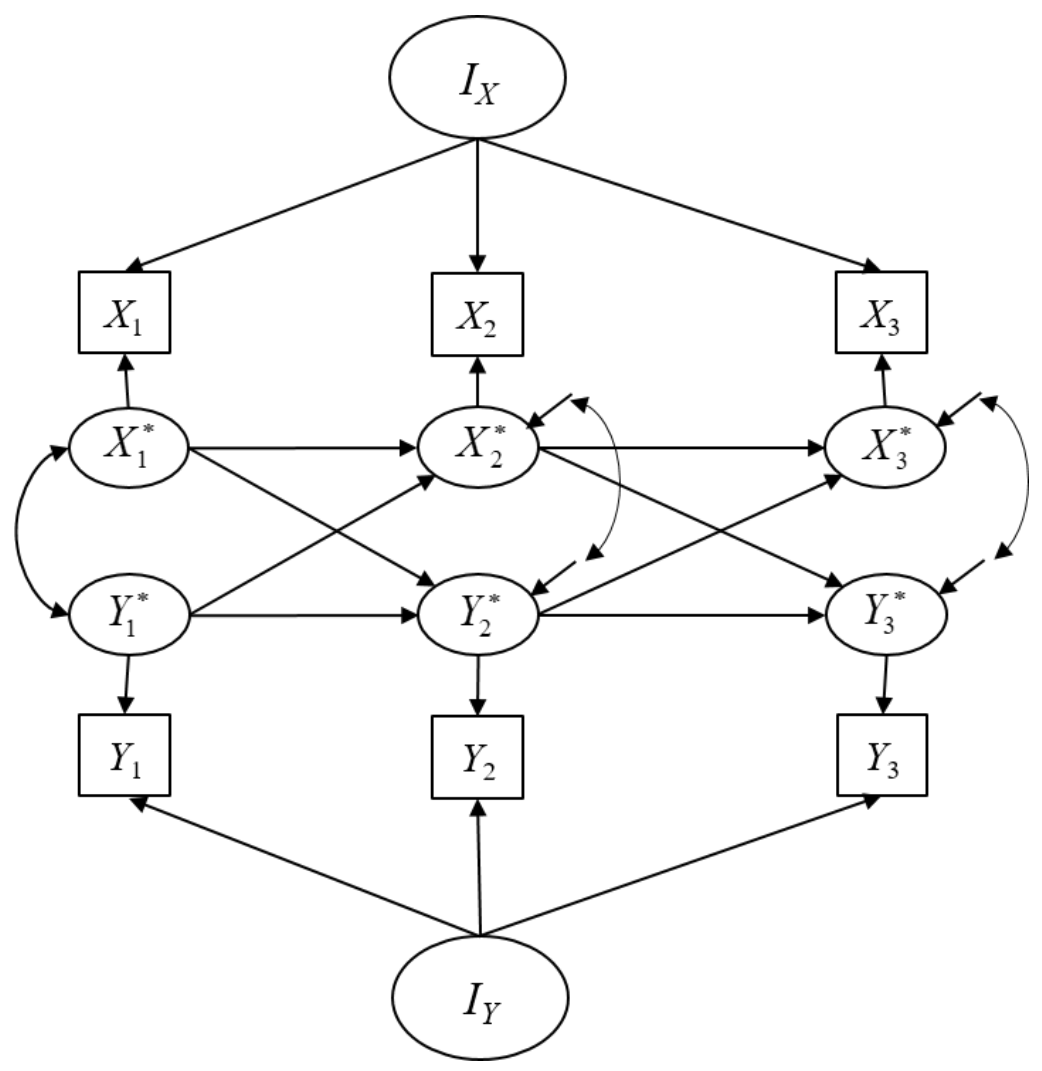

Figure 3. Path diagram of the random intercept cross-lagged panel model (RI-CLPM; see Hamaker et al., 2015) for three measurement waves.

The present article compares the CL1PM, CL2PM, and RI-CLPM from a causal inference perspective. We focus on the question under which assumptions these three methods provide unbiased estimates of the cross-lagged effect. It has been argued that the RI-CLPM (i.e., the coefficient $\beta_{Y_{t}^{*} X_{t-1}^{*}}$ ) provides a more conservative estimate of cross-lagged effects because it allows controlling for the effects of time-invariant and unobserved confounders. For example, Usami, Murayama, and Hamaker (2019, p. 652) argued that the RI-CLPM "can provide a stronger basis to infer causal effects by allowing for the presence of timeinvariant unobserved confounding variables" in estimating the cross-lagged effect. This reasoning is based on the idea that the stable trait factors $I_{X i}$ and $I_{Y i}$ account for the effects of time-invariant confounder variables. In the next section, we further investigate that claim and compare the RI-CLPM with the CL1PM and the CL2PM from a structural modeling perspective.

\section{Structural Modeling Perspective on Estimating Cross-Lagged Effects}

In the following, we use a structural modeling perspective (Pearl et al., 2016) to discuss the causal assumptions that are needed to identify the cross-lagged effect in the CL1PM, CL2PM, and RI-CLPM. We assume that two variables are measured across three measurement waves and are denoted as $Y_{i 1}, Y_{i 2}, Y_{i 3}$, and $X_{i 1}, X_{i 2}, X_{i 3}$, respectively. Fur- 
8

thermore, we allow for the presence of confounding variables $\mathbf{U}_{i}$ (e.g., physical health, social status, gender) that affect both variables across time. The assumed causal relationships between the variables are depicted in the structural model in Figure 4. In the following, we focus on the cross-lagged effect of $X_{i 2}$ on $Y_{i 3}$.

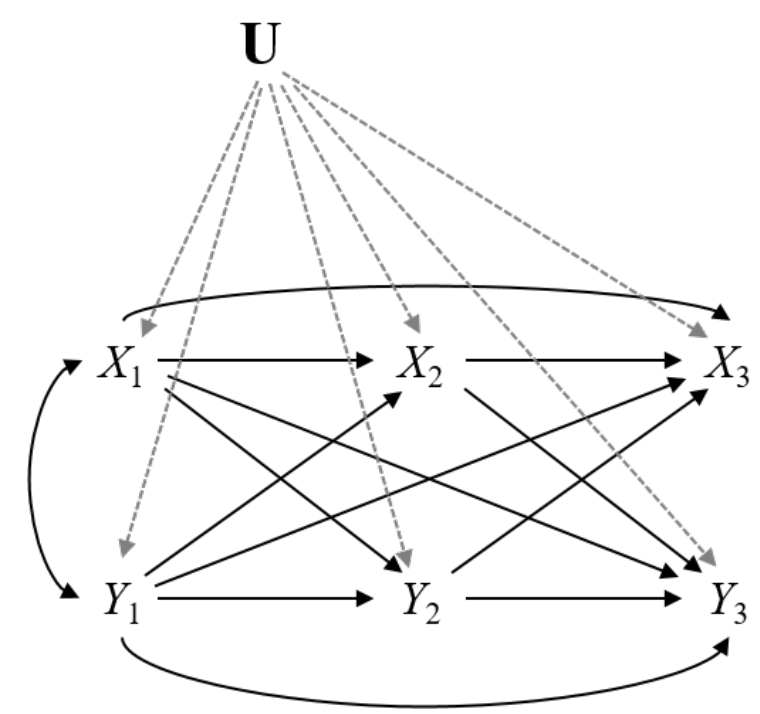

Figure 4. Structural model for a cross-lagged panel design with three measurement waves: $X$ (i.e., $X_{1}, X_{2}$, and $\left.X_{3}\right)$ and $Y\left(Y_{1}, Y_{2}\right.$, and $\left.Y_{3}\right)$ are repeatedly assessed at each wave, and $\mathbf{U}$ denotes a vector of covariates that affects $X$ and $Y$.

\subsection{Causal Definition of Cross-Lagged Effect}

The causal inference literature heavily draws on the potential outcome framework (Hernán \& Robins, 2020; Imbens \& Rubin, 2015; VanderWeele, 2015) to define a causal effect (i.e., the effect of $X_{i 2}$ on $\left.Y_{i 3}\right)$. We define a set of individuals $i(i=1, \ldots, N)$ and assume a continuous exposure variable $X_{i 2}$ taking values from a set $\mathcal{X}$ (Hirano \& Imbens, 2004; Vegetabile et al., 2021). We now assume that for each individual $i$, there exists a potential outcome $Y_{i 3}\left(x_{2}\right)$ for all $x_{2} \in \mathcal{X}$. The potential outcome $Y_{i 3}\left(x_{2}\right)$ can be interpreted as the outcome that would have resulted for individual $i$ if the exposure $X_{i 2}$ had been set to $x_{2}$ (e.g., by an intervention). We further assume that, for each individual, the observed outcome equals the potential outcome under the observed exposure level, that is, $Y_{i 3}=Y_{i 3}\left(x_{2}\right)$ if $X_{i 2}=x_{2}$. This assumption is also known as the consistency assumption and connects the potential outcomes to the observed data (Hernán \& Robins, 2020; VanderWeele, 2015). Note that all other potential outcomes for individual $i$, i.e., $Y_{i 3}\left(x_{2}^{\prime}\right)$ for all other $x_{2}^{\prime} \in \mathcal{X}$, are unobserved. The expectation of the potential outcomes is modeled as a linear function of the exposure values $x_{2}$

$$
\mathrm{E}\left(Y_{i 3}\left(x_{2}\right)\right)=\tau_{0}+\tau_{1} x_{2},
$$

where the parameter $\tau_{1}$ denotes the causal effect of increasing the exposure level by one unit from $x_{2}$ to $x_{2}+1$; that is, $\mathrm{E}\left(Y_{i 3}\left(x_{2}+1\right)-Y_{i 3}\left(x_{2}\right)\right)=\tau_{1}$. Equation (6) is also known as 
a marginal structural model (MSM), which specifies a model for the marginal mean of the potential outcomes as a function of the exposure, in which the effects of confounding variables have been removed (Daniel, Cousens, De Stavola, Kenward, \& Sterne, 2013; Hernán \& Robins, 2020). However, in many applications, the true regression function for Equation (6) might be nonlinear. In this case, the regression can be interpreted as the best linear approximation to the true and unknown regression function (Angrist \& Pischke, 2009; Berk et al., 2014).

It can be shown that the causal effect $\tau_{1}$ is identified under the ignorability assumption

$$
Y_{i 3}\left(x_{2}\right) \perp X_{i 2} \mid Y_{i 2}, Y_{i 1}, X_{i 1}, \mathbf{U}_{i}
$$

This assumption states that the potential outcomes are conditionally independent of the exposure $X_{i 2}$ given the previous values of $Y$ (i.e., $Y_{i 1}$ and $Y_{i 2}$ ), the previous value of $X_{i 1}$, and the confounders $\mathbf{U}_{i}$. This assumption is also labeled the unconfoundedness, conditional independence, or selection on observables assumption in the literature (Hernán \& Robins, 2020; Imbens, 2004; Morgan \& Winship, 2014). The goal is then to estimate the causal effect $\tau_{1}$ from the data $\left(Y_{i 3}, X_{i 2}, Y_{i 2}, Y_{i 1}, X_{i 1}, \mathbf{U}_{i}\right)$. If the ignorability assumption holds, the expectation of the potential outcomes can be identified as follows:

$$
\begin{aligned}
& \mathrm{E}\left[Y_{i 3}\left(x_{2}\right)\right] \\
= & \int \mathrm{E}\left(Y_{i 3} \mid X_{i 2}=x_{2}, X_{i 1}=x_{1}, Y_{i 2}=y_{2}, Y_{i 1}=y_{1}, \mathbf{U}_{i}=\boldsymbol{u}\right) f\left(x_{2}, x_{1}, y_{2}, y_{1}, \boldsymbol{u}\right) \mathrm{d} x_{1} \mathrm{~d} y_{2} \mathrm{~d} y_{1} \mathrm{~d} \boldsymbol{u}
\end{aligned}
$$

where $f$ denotes the joint density of $X_{i 2}, X_{i 1}, Y_{i 2}, Y_{i 1}$, and $\mathbf{U}_{i}$. In other words, the expected values of the potential outcomes can be determined by averaging the conditional expectation of the outcome given the covariates and the exposure level across the covariate distribution. Thus, the ignorability assumption ensures that the causal effect in Equation (7) can be estimated from the observed data:

$$
\tau_{1}=\frac{\left.\left.\operatorname{Cov}\left[\mathrm{E}\left(Y_{i 3}\left(X_{i 2}\right)\right), X_{i 2}\right)\right]\right)}{\operatorname{Var}\left(X_{i 2}\right)}=\frac{\mathrm{E}\left[\mathrm{E}\left(Y_{i 3}\left(X_{i 2}\right)\right) X_{i 2}\right]-\mathrm{E}\left[Y_{i 3}\left(X_{i 2}\right)\right] \mathrm{E}\left(X_{i 2}\right)}{\operatorname{Var}\left(X_{i 2}\right)},
$$

where the expectation of the potential outcomes is plugged into the formula for a simple regression slope. Again, note that if the true regression function $x_{2} \mapsto \mathrm{E}\left(Y_{i 3}\left(x_{2}\right)\right)$ is nonlinear, $\tau_{1}$ can be interpreted as the slope of the best linear approximation of the regression function (Angrist \& Pischke, 2009).

It should be emphasized that ignorability is a strong assumption that should not be taken lightly ${ }^{1}$. In practical applications, the ignorability assumption has two aspects. First, it is assumed that all relevant covariates (that are needed to fulfill the ignorability assumption in Equation (7)) are observed. It is vital that this aspect of the ignorability assumption cannot be empirically tested and needs to be justified by substantive knowledge (Aronow \& Miller, 2019). Second, it requires that the relationship between the covariates $\left(Y_{i 2}, Y_{i 1}, X_{i 1}\right.$, and $\left.\mathbf{U}_{i}\right)$ and the exposure $\left(X_{i 2}\right)$ is correctly specified in a given application.

\footnotetext{
${ }^{1}$ Usually, a second assumption is added (positivity assumption) which states that in the population, the density of observing any exposure level given the covariates is positive. This assumption implies that there exists sufficient overlap in the covariate distributions between the different exposure levels under consideration.
} 
This second aspect implies that not only all relevant covariates are observed but that also the functional form of the relationship is correctly specified in the analysis models (e.g., squared terms of predictors are included if quadratic effects exist).

In order to simplify the discussion, we assume in the following that all observed variables are linearly related and that only one time-invariant confounder $U_{i}$ exists. The structural model in Figure 4 can then be interpreted as a potential data-generating model in which the equation for the outcome $Y_{i 3}$ is given as follows

$$
Y_{i 3}=\gamma_{Y_{3}}+\gamma_{Y_{3} Y_{2}} Y_{i 2}+\gamma_{Y_{3} Y_{1}} Y_{i 1}+\gamma_{Y_{3} X_{2}} X_{i 2}+\gamma_{Y_{3} X_{1}} X_{i 1}+\gamma_{Y_{3} U} U_{i}+\varepsilon_{Y_{3} i}
$$

Our main goal is to estimate the effect $\gamma_{Y_{3} X_{2}}$ of exposure $X_{i 2}$ on the outcome $Y_{i 3}$. It can now be shown that under the assumed data-generating model, the effect $\gamma_{Y_{3} X_{2}}$ provides the causal effect $\tau_{1}$. Using the relationship in Equation $(10)^{2}$, we insert Equation (8) into Equation (9)

$$
\tau_{1}=\frac{\left.\left.\operatorname{Cov}\left[\mathrm{E}\left(Y_{i 3}\left(X_{i 2}\right)\right), X_{i 2}\right)\right]\right)}{\operatorname{Var}\left(X_{i 2}\right)}=\frac{\operatorname{Cov}\left[\gamma_{Y_{3} X_{2}} X_{i 2}, X_{i 2}\right]}{\operatorname{Var}\left(X_{i 2}\right)}=\gamma_{Y_{3} X_{2}}
$$

In the second equation, we use that the covariance remains unaltered by adding a constant to a variable. This also illustrates that the cross-lagged effect $\gamma_{Y_{3} X_{2}}$ of the CL2PM (i.e., the traditional CLPM with lag-2 effects) provides an unbiased estimate of the causal effect $\tau_{1}$ when all relevant covariates (i.e., $Y_{i 2}, Y_{i 1}, X_{i 1}$, and $U_{i}$ ) are included.

\subsection{A Formal Analysis of Controlling for Confounders in the RI-CLPM}

We now present a formal analysis of the potential of the RI-CLPM to control for timeinvariant confounder variables (see Usami, Murayama, \& Hamaker, 2019). To this end, we assume a lag-1 model with time-invariant confounder $U_{i}$ and three waves of measurements as a structural model (see Figure 5). For simplicity, we further assume that the variables are mean centered at each wave. Under the assumption of linear effects, the model for the outcome $Y_{i 3}$ is given as follows

$$
Y_{i 3}=\gamma_{Y_{3} Y_{2}} Y_{i 2}+\gamma_{Y_{3} X_{2}} X_{i 2}+\gamma_{Y_{3} U} U_{i}+\varepsilon_{Y_{3} i}
$$

Our main goal is to estimate the cross-lagged effect $\gamma_{Y_{3} X_{2}}$ of $X_{i 2}$ on $Y_{i 3}$ in the presence of an unobserved confounding variable $U_{i}$. As clarified in the previous section (see Equation (6)), this coefficient can be interpreted as the effect of increasing the exposure $X_{i 2}$ by one unit from $x_{2}$ to $x_{2}+1$ on the expected potential outcomes $\mathrm{E}\left(Y_{i 3}\left(x_{2}\right)\right)$. In our discussion, we apply the RI-CLPM for mean centered variables

$$
\begin{aligned}
X_{i t} & =U_{X i}+X_{i t}^{*} \\
Y_{i t} & =U_{Y i}+Y_{i t}^{*} \\
Y_{i t}^{*} & =\beta_{Y_{t}^{*} Y_{t-1}^{*}} Y_{i, t-1}^{*}+\beta_{Y_{t}^{*} X_{t-1}^{*}} X_{i, t-1}^{*}+\delta_{Y_{t} i}
\end{aligned}
$$

\footnotetext{
${ }^{2}$ We used the fact that under the structural model in Equation (10) averaging the conditional expectation of the outcome given the covariates in Equation (8) across the covariate distribution gives $\mathrm{E}\left(Y_{i 3}\left(X_{i 2}\right)\right)=$ $\gamma_{Y_{3}}+\gamma_{Y_{3} Y_{2}} \mathrm{E}\left(Y_{i 2}\right)+\gamma_{Y_{3} Y_{1}} \mathrm{E}\left(Y_{i 1}\right)+\gamma_{Y_{3} X_{2}} X_{i 2}+\gamma_{Y_{3} X_{1}} \mathrm{E}\left(X_{i 1}\right)$.
} 


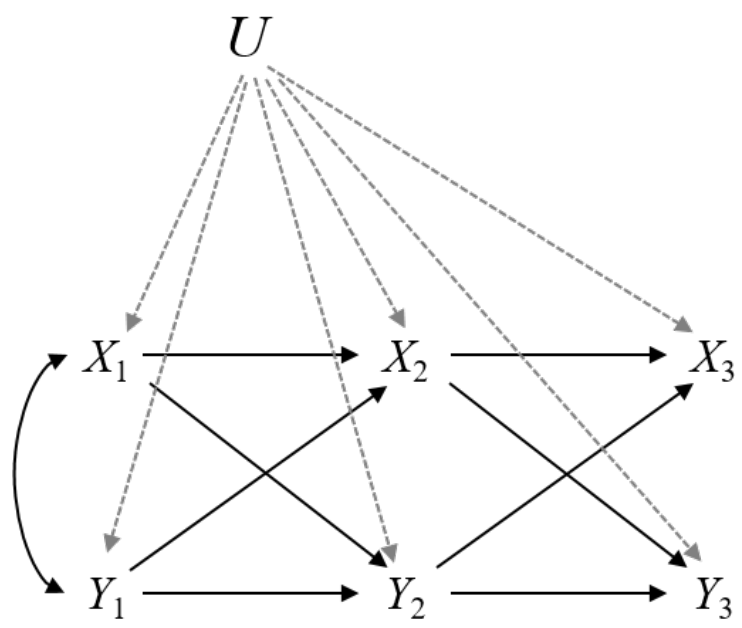

Figure 5. Structural model with only lag-1 effects and a confounder $U$ that affects $X$ and $Y$.

The RI-CLPM with $Y_{i 3}$ as outcome variable can be rewritten in one equation as follows

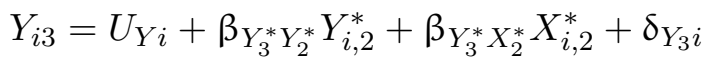

It needs to be emphasized that the random intercept (i.e., $U_{Y i}$ ), and the decomposed withinperson predictors (i.e., $Y_{i 2}^{*}$ and $X_{i 2}^{*}$ ) in the RI-CLPM in Equation (16) will, in general, be different from the confounder (i.e., $U_{i}$ ) and the undecomposed predictors (i.e., $Y_{i 2}$ and $X_{i 2}$ ) in the structural model (see Equation (12)).

We now clarify the conditions under which $\beta_{Y_{3}^{*} X_{2}^{*}}$ in the RI-CLPM (see Equation (16)) provides an unbiased estimate of the cross-lagged effect $\gamma_{Y_{3} X_{2}}$ in the structural model (see Equation (12)). To this end, we write the linear projection of the confounder $U_{i}$ on the two stable trait components $U_{Y i}$ and $U_{X i}$ (see Wooldridge, 2010):

$$
U_{i}=\alpha_{U_{Y}} U_{Y i}+\alpha_{U_{X}} U_{X i}+V_{i}
$$

where $\alpha_{U_{Y}}$ and $\alpha_{U_{X}}$ describe the (linear) relationship of $U_{i}$ with $U_{Y i}$ and $U_{X i}$, and $V_{i}$ is uncorrelated with $U_{Y i}$ and $U_{X i}$ and has zero mean. Using the linear projection in Equation (17) and the decompositions in Equations (13) and (14), we rewrite the structural model in Equation (12) as follows

$$
\begin{aligned}
Y_{i t}= & \gamma_{Y_{3} Y_{2}}\left(U_{Y i}+Y_{i 2}^{*}\right)+\gamma_{Y_{3} X_{2}}\left(U_{X i}+X_{i 2}^{*}\right)+\gamma_{Y_{3} U}\left(\alpha_{U_{Y}} U_{Y i}+\alpha_{U_{X}} U_{X i}+V_{i}\right)+\varepsilon_{Y_{3} i} \\
= & \left(\gamma_{Y_{3} X_{2}}+\gamma_{Y_{3} U} \alpha_{U_{X}}\right) U_{X i}+\left(\gamma_{Y_{3} Y_{2}}+\gamma_{Y_{3} U} \alpha_{U_{Y}}\right) U_{Y i} \\
& \quad+\gamma_{Y_{3} Y_{2}} Y_{i 2}^{*}+\gamma_{Y_{3} X_{2}} X_{i 2}^{*}+\gamma_{Y_{3} U} V_{i}+\varepsilon_{Y_{3} i}
\end{aligned}
$$

where $V_{i}$ and $\varepsilon_{Y_{3} i}$ are uncorrelated residual components with zero means. By comparing coefficients between the rewritten structural model in Equation (18) and the RI-CLPM in Equation (16), we now obtain: $\gamma_{Y_{3} X_{2}}+\gamma_{Y_{3} U} \alpha_{U_{X}}=0, \gamma_{Y_{3} Y_{2}}+\gamma_{Y_{3} U} \alpha_{U_{Y}}=1, \gamma_{Y_{3} Y_{2}}=\beta_{Y_{3}^{*} Y_{2}^{*}}$, 
and $\gamma_{Y_{3} X_{2}}=\beta_{Y_{3}^{*} X_{2}^{*}}$. Based on these relationships, we can state the following conditions under which $\beta_{Y_{3}^{*} X_{2}^{*}}$ of the RI-CLPM provides an unbiased estimate of the cross-lagged effect $\gamma_{Y_{3} X_{2}}$ in the structural model:

$$
\begin{aligned}
\alpha_{U_{Y}} & =\frac{\left(1-\beta_{Y_{3}^{*} Y_{2}^{*}}\right)}{\gamma_{Y_{3} U}} \\
\alpha_{U_{X}} & =-\frac{\beta_{Y_{3}^{*} X_{2}^{*}}}{\gamma_{Y_{3} U}}
\end{aligned}
$$

Thus, the potential of the RI-CLPM to account for unobserved confounding is limited by the fact that very restrictive conditions need to be met for the effects of the confounder variable $U_{i}$. These conclusions contradict the formal analysis of the causal implications of the RI-CLPM presented by Usami, Murayama, and Hamaker (2019). Their formal analysis provided extended identifiability assumptions that should allow the RI-CLPM to "account for unobserved confounding caused by time-invariant factors" (p. 650). However, in their structural model (see Equation 24 in Usami, Murayama, \& Hamaker, 2019), they implausibly assumed that the random intercept for $Y$ equals the unobserved confounder (i.e., $\left.U_{i}=U_{Y i}\right)$. It can be shown that this assumption only holds true if the stable trait factors are perfectly negatively correlated, that is, $\operatorname{Cor}\left(U_{Y i}, U_{X i}\right)=-1$ (see the Appendix for a more detailed explanation). This does not seem plausible for most applications of the RI-CLPM with panel data.

Alternatively, to better align the RI-CLPM with the potential outcome approach, one can redefine the causal effect of interest. Recently, Usami (2020; see also Gische, West, \& Voelkle, 2021) introduced the idea of within-person variability score-based causal inference $^{3}$. In this approach, the potential outcomes are defined at the within-person level; that is, $Y_{i 3}^{*}\left(x_{2}^{*}\right)$. A corresponding marginal structural model would be defined as

$$
\mathrm{E}\left(Y_{i 3}^{*}\left(x_{2}^{*}\right)\right)=\gamma_{Y_{3} X_{2}} x_{2}^{*}
$$

In this case, the cross-lagged effect $\beta_{Y_{3}^{*} X_{2}^{*}}$ of the RI-CLPM in Equation (16) would provide unbiased estimates of $\gamma_{Y_{3} X_{2}}$. However, it needs to be emphasized that this would also redefine the causal estimand (i.e., the target of inference). In the traditional definition of a causal effect in the potential outcome approach, the causal effect describes the effect of increasing the exposure $X_{2}$ one unit from $x_{2}$ to $x_{2}+1$ on $Y_{i 3}\left(x_{2}\right)$, and this is also the definition of a causal effect that Usami, Murayama, and Hamaker (2019, see p. 650) used. When the marginal structural model is based on the decomposed variables as in Equation (20), the causal effect would be given by a unit change around the individual person mean. It is essential to understand that these two definitions of the causal effect imply fundamentally different targets of causal inference.

In the within-subject perspective of the RI-CLPM, the causal effect only targets short-term influences of a variable $X$ on a variable $Y$ because only the temporal deviations

\footnotetext{
${ }^{3}$ In this paper, Usami (2020) emphasized that the RI-CLPM could be better integrated in the causal inference literature: "Despite its popularity and theoretical appeal, the concepts of within-person effects in the RI-CLPM have not been fully characterized in the causal inference literature. This might be partly because psychometricians have used the terms stable traits and within-person relations in ambiguous ways for different models (Usami, Murayama, \& Hamaker, 2019)."
} 
from the stable parts (i.e., fluctuations around the individual person means) are analyzed. By contrast, in the CLPM analysis strategy, the causal effect targets systematic changes of persons that can also explain why differences between persons evolve across time (see Hoffman, 2015). For example, let us assume one is interested in estimating the positive effect of academic self-concept on school achievement (e.g., Ehm et al., 2019; Marsh et al., 2018). In this case, the cross-lagged effect of the CLPM addresses the question of whether students who have higher self-concept scores (compared to other students) at time point $t$ are also likely to show higher achievement (compared to other students) at time point $t+1$. By contrast, the within-person cross-lagged effect of the RI-CLPM captures whether students who have higher deviations from their (long-term) average self-concept score at time point $\mathrm{t}$ are more likely to show higher deviations from their (long-term) average achievement score at time point $t+1$. In our view, the cross-lagged effect of the CLPM is of more interest in this example, particularly if researchers are interested in understanding the potential causes (e.g., potential interventions that enhance students' self-concept) that make students different from other students in terms of their achievement - and not only different from their average achievement level. We believe that the within-person effects of the RI-CLPM are more appropriate for studies with shorter time lags between assessments (e.g., intensive longitudinal designs; see Bolger \& Laurenceau, 2013; Hoffman, 2015) that are not concerned about systematic changes between persons but explicitly focus on questions about shortterm effects that capture within-person dynamics (e.g., daily fluctuations around a stable person mean).

\section{Estimation of Cross-Lagged Effects under Different Data-Generating Models}

We now use simulated data to illustrate further the conditions under which the CL1PM, CL2PM, and RI-CLPM provide unbiased estimates of the cross-lagged effect. We distinguish three different scenarios for a cross-lagged panel design with three measurement waves and two variables (i.e., $X$ and $Y$ ). In Scenario A, we assumed that the true data-generating model was a RI-CLPM. In Scenario B, we assumed the true model was a CL2PM. In Scenario C, the data were generated by a CL1PM and a confounder variable $U$. For each scenario, we present the results for six different data conditions generated under different parameters of the data-generating models. As we were only interested in the bias of the parameter estimates, we simulated only one large data set $(N=10000)$ for each data condition. In all scenarios, the variables (i.e., $X_{1}, X_{2}, X_{3}, Y_{1}, Y_{2}, Y_{3}$, and $U$ ) were standardized with zero means and variances of one. Our discussion focuses on the estimation of the (lag-1) cross-lagged effect of $X_{2}$ on $Y_{3}$. The $\mathrm{R}$ and lavaan (Rosseel, 2012) code for the data-generating models and the different analysis models is provided in Supplements S1, S2, and S3 at https://osf.io/s9bnv/.

\subsection{Scenario A: RI-CLPM as the Data-Generating Model}

In Scenario A, we assumed that the RI-CLPM is the data-generating model with a true cross-lagged effect of $\beta_{Y_{3}^{*} X_{2}^{*}}=0.20$. We manipulated the random-intercept variance of $X$ (i.e., $\phi_{I X}=.3, .5$, and .7) and the correlation of the random intercepts of $X$ and $Y$ (i.e., $\rho_{I X Y}=.5$, and .7), resulting in six different data conditions. We analyzed the six data sets 
with the RI-CLPM, CL1PM, and CL2PM (see Table 1) ${ }^{4}$. As expected, the estimates of the RI-CLPM were unbiased in all six conditions. However, the estimates of the CL1PM were consistently too small and underestimated the true magnitude of the cross-lagged effect, particularly when the correlation between the random intercepts was .5. For example, in data condition A2 (i.e., $\phi_{I X}=.5$ and $\rho_{I X Y}=.5$ ), the estimate provided by the CL1PM was $50 \%$ smaller than the true coefficient (.10 vs. .20). These findings replicated the results from Hamaker et al. (2015) and other simulation studies (Usami, Todo, \& Murayama, 2019) that the CL1PM can produce distorted estimates of cross-lagged effects when the true data-generating model is a RI-CLPM.

Table 1

Results for Scenario A: True Model is the Random Intercept Cross-Lagged Panel Model (RICLPM). Estimates for the Cross-Lagged Effect for the CL1PM, CL2PM, and RI-CLPM.

\begin{tabular}{ccccccc}
\hline Dataset & $\phi_{I X}$ & $\rho_{I X Y}$ & $\beta_{Y_{3}^{*} X_{2}^{*}}$ & CL1PM & CL2PM & RI-CLPM \\
\hline A1 & 0.3 & 0.5 & 0.20 & $\mathbf{0 . 1 1}$ & $\mathbf{0 . 1 6}$ & 0.20 \\
A2 & 0.5 & 0.5 & 0.20 & $\mathbf{0 . 1 0}$ & $\mathbf{0 . 1 5}$ & 0.20 \\
A3 & 0.7 & 0.5 & 0.20 & $\mathbf{0 . 0 9}$ & $\mathbf{0 . 1 4}$ & 0.20 \\
A4 & 0.3 & 0.7 & 0.20 & $\mathbf{0 . 1 5}$ & $\mathbf{0 . 1 8}$ & 0.20 \\
A5 & 0.5 & 0.7 & 0.20 & $\mathbf{0 . 1 5}$ & $\mathbf{0 . 1 7}$ & 0.20 \\
A6 & 0.7 & 0.7 & 0.20 & $\mathbf{0 . 1 5}$ & $\mathbf{0 . 1 7}$ & 0.20 \\
\hline
\end{tabular}

Note. CL1PM $=$ cross-lagged panel model with lag-1 effects; CL2PM $=$ cross-lagged panel model with lag-2 effects; RI-CLPM $=$ random intercept cross-lagged panel model. $\phi_{I X}=$ true variance of the random intercepts of $X ; \rho_{I X Y}=$ true correlation of the random intercepts of $X$ and $Y$; $\beta_{Y_{3}^{*} X_{2}^{*}}=$ true cross-lagged effect of $X_{2}^{*}$ on $Y_{3}^{*}$ in the RI-CLPM. Absolute biases for the estimates of the cross-lagged effect larger than .02 are printed in bold.

In addition, the CL2PM that includes the lag-2 effects (i.e., effects of $X_{1}$ and $Y_{1}$ on $X_{3}$ and $Y_{3}$ ) produced estimates that underestimated the true magnitude of the crosslagged effect. The bias for the CL2PM was smaller but followed a similar pattern as the bias of the CL1PM (i.e., smaller bias with a larger correlation of the random intercepts). This is an important finding because it shows that even a saturated model with a perfect fit (i.e., the CL2PM perfectly reproduced the covariance structure of the simulated data) did not guarantee that the resulting parameter estimates are unbiased. However, it also needs to be emphasized that Scenario A (i.e., RI-CLPM is the data-generating model) implicitly assumes that either the causal effect of interest is defined at the within-person level (see Equation (20)) or that an unobserved confounder $U$ exists that fulfills the restrictive conditions in Equation (19).

\subsection{Scenario B: CL2PM as the Data-Generating Model}

In Scenario B, we assumed that the data were generated by a CL2PM (see Equation (2)). More specifically, we assumed a stationary process that fulfills a cross-lagged panel

\footnotetext{
${ }^{4}$ The regression coefficients were freely estimated and no stationarity constraints were introduced. The RI-CLPM had one degree of freedom $(d f=1)$, the CL1PM four degrees of freedom, and the CL2PM was saturated $(d f=0)$.
} 
model with lag-2 effects (i.e., the lag- 1 covariances were assumed to be constant for each data condition). We manipulated the lag-2 covariances by specifying different values for the lag-2 cross-lagged effects (i.e., $\beta_{Y_{3} X_{1}}$ ), and the lag-2 autoregressive effects (i.e., $\beta_{Y_{3} Y_{1}}$ ). This resulted in six different data conditions in which the synchronous and lag-1 correlations were constant, but the lag-2 correlations differed.

As expected, the CL2PM that includes lag-2 effects provided unbiased estimates of the cross-lagged effect under all data conditions in this scenario (see Table 2). The CL1PM produced positively biased estimates and overestimated the size of the true cross-lagged effect. For example, in condition B2 with small lag- 2 cross-lagged effects (i.e., $\beta_{Y_{3} X_{1}}=.01$ ), and a substantial lag-2 autoregressive effect (i.e., $\beta_{Y_{3} Y_{1}}=.32$ ), the true cross-lagged effect is overestimated by $100 \%$ in the CL1PM (.20 vs. .10). This illustrates that the estimates of the CL1PM can be strongly distorted by the presence of delayed effects that are not adequately captured by the lag-1 effects (VanderWeele et al., 2020; see also Marsh et al., 2018). Note that the estimated cross-lagged effects in the CL1PM did not change across the data conditions because the lag- 1 correlations were fixed, and only the lag- 2 correlations were manipulated.

Table 2

Results for Scenario B: True Model is the Cross-Lagged Panel Model with Lag-2 Effects (CL2PM). Estimates for the Cross-Lagged Effect for the CL1PM, CL2PM, and RI-CLPM.

\begin{tabular}{crccccc}
\hline Dataset & $\beta_{Y_{3} X_{1}}$ & $\beta_{Y_{3} Y_{1}}$ & $\beta_{Y_{3} X_{2}}$ & CL1PM & CL2PM & RI-CLPM \\
\hline B1 & 0.08 & 0.27 & 0.10 & $\mathbf{0 . 2 0}$ & 0.10 & $\mathbf{0 . 0 2}$ \\
B2 & 0.01 & 0.32 & 0.10 & $\mathbf{0 . 2 0}$ & 0.10 & $-\mathbf{0 . 0 4}$ \\
B3 & 0.04 & 0.29 & 0.12 & $\mathbf{0 . 2 0}$ & 0.12 & $\mathbf{0 . 0 8}$ \\
B4 & -0.01 & 0.33 & 0.12 & $\mathbf{0 . 2 0}$ & 0.12 & $\mathbf{0 . 0 3}$ \\
B5 & 0.03 & 0.27 & 0.14 & $\mathbf{0 . 2 0}$ & 0.14 & 0.13 \\
B6 & 0.00 & 0.30 & 0.14 & $\mathbf{0 . 2 0}$ & 0.14 & 0.14 \\
\hline
\end{tabular}

Note. CL1PM $=$ cross-lagged panel model with lag-1 effects; CL2PM $=$ cross-lagged panel model with lag-2 effects; RI-CLPM = random intercept cross-lagged panel model. $\beta_{Y_{3} X_{1}}=$ true lag-2 cross-lagged effect of $X_{1}$ on $Y_{3} ; \beta_{Y_{3} Y_{1}}=$ true lag-2 autoregressive effect of $Y_{1}$ on $Y_{3} ; \beta_{Y_{3} X_{2}}=$ true lag-1 cross-lagged effect of $X_{2}$ on $Y_{3}$. Absolute biases for the estimates of the lag-1 cross-lagged effect larger than .02 are printed in bold.

Most importantly, the performance of the RI-CLPM strongly depended on the size of the lag-2 effects, and the RI-CLPM tended to underestimate the magnitude of the true crosslagged effect. In condition B2 (i.e., $\beta_{Y_{3} X_{1}}=.01$, and $\beta_{Y_{3} Y_{1}}=.32$ ), the estimate provided by the RI-CLPM even had a different sign than the true cross-lagged effect ( -.04 vs. .10). In addition, the bias of the RI-CLPM completely vanished when no lag- 2 cross-lagged effect was present in the condition B6 (i.e., $\beta_{Y_{3} X_{1}}=0$ ).

Overall, the findings in scenario $\mathrm{B}$ are in line with a pattern of results that we have also seen in empirical applications of the different cross-lagged panel models: the CL1PM produces larger cross-lagged effects than the RI-CLPM, and the estimates of the CL2PM are between the two approaches (e.g., Ehm et al., 2019). In addition, Scenario B confirms findings from the methodological literature that the CL1PM can positively bias estimates 
of cross-lagged effects because it provides insufficient control for confounding due to the lag-2 effects. The stable latent trait factors of the RI-CLPM capture these additional lag-2 effects. However, in general, they do not appropriately control for the confounding due to previous measures of the exposure and the outcome, resulting in overadjusted estimates of cross-lagged effects.

It needs to be emphasized that the decision between the CL2PM and the RI-CLPM cannot be made on the basis of model fit. The RI-CLPM showed a very good fit in all six data conditions (SRMR <.02; see West, Taylor, \& Wu, 2012), whereas the CL2PM was saturated and provided a perfect fit. Thus, researchers would accept both models as an adequate description of the observed data and would need to rely on other criteria for choosing between different estimates of the cross-lagged effect. We come back to this issue in the final Discussion section.

\subsection{Scenario C: CL1PM with Confounder $U$ as the Data-Generating Model}

In Scenario C, we illustrate the potential of the RI-CLPM to control for the presence of an unobserved confounder variable $U$. We assumed that the data were generated by a CL1PM with an additional (time-invariant) confounder variable $U$ that affects all six observed variables. We fixed the true value of the cross-lagged effect $\left(\beta_{Y_{3} X_{1}}=.20\right)$ and manipulated the effect of $U$ on the observed variables by assuming that the correlation between $U$ and $Y$ decreased by a factor of $.5\left(\rho_{Y_{1} U}=.50, \rho_{Y_{2} U}=.25\right.$, and $\left.\rho_{Y_{3} U}=.13\right)$, was constant across time $\left(\rho_{Y_{1} U}=\rho_{Y_{2} U}=\rho_{Y_{3} U}=.50\right)$, or increased by a factor of $2\left(\rho_{Y_{1} U}=.04\right.$, $\rho_{Y_{2} U}=.09$, and $\left.\rho_{Y_{3} U}=.18\right)$ or $3\left(\rho_{Y_{1} U}=.05, \rho_{Y_{2} U}=.15\right.$, and $\left.\rho_{Y_{3} U}=.47\right)$. In addition, we assumed that the correlation between $X$ and $U$ decreased by a factor of $.5\left(\rho_{X_{1} U}=.30\right.$, $\rho_{X_{2} U}=.15$, and $\left.\rho_{X_{3} U}=.08\right)$ or was constant across time $\left(\rho_{X_{1} U}=\rho_{X_{2} U}=\rho_{X_{3} U}=\right.$ $.30)$. Overall, this resulted in six data conditions. To separate the issue of lag-2 effects (see Scenario B) from the treatment of (unobserved) confounders, we did not specify lag-2 effects in the data-generating model. Again, we analyzed the six data sets with the RICLPM, CL1PM, and CL2PM and specified RI-CLPM and CL2PM models that included the confounder $U$ (labeled as RI-CLPMU and CL2PMU) ${ }^{5}$.

Table 3 shows the results for the six data conditions. As expected, the models that included the confounder variable $U$ (CL2PMU and RI-CLPMU) produced unbiased estimates of the cross-lagged effect. By contrast, the models that do not include the confounder (CL1PM, CL2PM, and RI-CLPM) can produce positively or negatively biased estimates of the cross-lagged effect, depending on the effect of the confounding variable. For example, in condition $\mathrm{C} 6$ with a confounding variable that had an increasing effect across time, all three models provided positively biased estimates of the cross-lagged effect (CL1PM: .30, CL2PM: .26, RI-CLPM: .24). Most importantly, the estimates of the RI-CLPM were only unbiased in data conditions in which the correlations of the confounder with $X$ and $Y$ were constant across time (condition C4), or if the correlations of $X$ and $Y$ with the confounder decreased by the same factor across time (condition $\mathrm{C} 1$ ). This finding illustrates again that the RI-CLPM only allows controlling for unobserved confounding under very restrictive conditions (see Equation (19)).

\footnotetext{
${ }^{5}$ We regressed the six observed variables on the confounder variable $U$, resulting in a model with $d f=1$ for the RI-CLPMU (see Mulder \& Hamaker, 2021), and a saturated model for CL2PMU.
} 
A CRITIQUE OF THE RANDOM INTERCEPT CROSS-LAGGED PANEL MODEL 17

Table 3

Results for Scenario C: True Model is the Cross-Lagged Panel Model with Lag-1 Effects and a Confounder U. Estimates for the Cross-Lagged Effect for the CL1PM, CL2PM, CL2PMU, $R I-C L P M$, and RI-CLPMU.

\begin{tabular}{ccccccccccccc}
\hline Dataset & $\rho_{Y_{1} U}$ & $\rho_{Y_{2} U}$ & $\rho_{Y_{3} U}$ & $\rho_{X_{1} U}$ & $\rho_{X_{2} U}$ & $\rho_{X_{3} U}$ & $\beta_{Y_{3} X_{2}}$ & CL1PM & CL2PM & CL2PMU & RI-CLPM & RI-CLPMU \\
\hline C1 & 0.50 & 0.25 & 0.13 & 0.30 & 0.15 & 0.08 & 0.20 & 0.20 & 0.20 & 0.20 & 0.20 & 0.20 \\
C2 & 0.50 & 0.25 & 0.12 & 0.30 & 0.30 & 0.30 & 0.20 & 0.19 & 0.19 & 0.20 & $\mathbf{0 . 1 2}$ & 0.20 \\
C3 & 0.51 & 0.50 & 0.51 & 0.30 & 0.15 & 0.07 & 0.20 & $\mathbf{0 . 1 7}$ & $\mathbf{0 . 1 7}$ & 0.20 & $\mathbf{0 . 1 7}$ & 0.20 \\
C4 & 0.50 & 0.50 & 0.50 & 0.30 & 0.30 & 0.30 & 0.20 & 0.21 & 0.20 & 0.20 & 0.20 & 0.20 \\
C5 & 0.04 & 0.09 & 0.18 & 0.30 & 0.15 & 0.07 & 0.20 & 0.21 & 0.20 & 0.20 & $\mathbf{0 . 1 8}$ & 0.20 \\
C6 & 0.05 & 0.15 & 0.47 & 0.30 & 0.31 & 0.30 & 0.20 & $\mathbf{0 . 3 0}$ & $\mathbf{0 . 2 6}$ & 0.20 & $\mathbf{0 . 2 4}$ & 0.20 \\
\hline
\end{tabular}

Note. CL1PM $=$ cross-lagged panel model with lag-1 effects; CL2PM $=$ cross-lagged panel model with lag-2 effects; CL2PMU = cross-lagged panel model with lag-2 effects and confounder $U$; RI-CLPM $=$ random intercept cross-lagged panel model; RI-CLPMU $=$ random intercept crosslagged panel model and confounder $U . \rho_{Y_{1} U}=$ true correlation between $Y_{1}$ and $U ; \rho_{Y_{2} U}=$ true correlation between $Y_{2}$ and $U ; \rho_{Y_{3} U}=$ true correlation between $Y_{3}$ and $U ; \rho_{X_{1} U}=$ true correlation between $X_{1}$ and $U ; \rho_{X_{2} U}=$ true correlation between $X_{2}$ and $U ; \rho_{X_{3} U}=$ true correlation between $X_{3}$ and $U ; \beta_{Y_{3} X_{2}}=$ true lag-1 cross-lagged effect of $X_{2}$ on $Y_{3}$. Absolute biases for the estimates of the lag-1 cross-lagged effect larger than .02 are printed in bold.

\subsection{Summary}

The main findings of the simulations can be summarized as follows. First, when the data were generated by a RI-CLPM (Scenario A), only the RI-CLPM produced unbiased estimates of the cross-lagged effects in all conditions (see Hamaker et al., 2015). However, when the CL2PM was the true model (Scenario B), the estimates of the RI-CLPM were biased in many conditions, indicating that the stable trait factors did not appropriately control for the lag-2 effects in the CL2PM. In practical applications with real data, the decision between the RI-CLPM and the CL2PM cannot be guided by model fit because both models will often provide a very good description of the data. Second, when a confounder variable is present, and the true model is the CL1PM (Scenario C), all approaches produced unbiased estimates of the cross-lagged effect if the confounder variable was included in the model. Third, in the presence of an unobserved confounder variable, all three approaches were limited in their potential to recover the true cross-lagged effect. This was expected for the CL1PM and CL2PM because these models are based on a selection-on-observables approach that assumes that all relevant covariates are measured. However, the results did not support the claim that the RI-CLPM provides a stronger control for confounding variables when estimating cross-lagged effects (Usami, Murayama, \& Hamaker, 2019).

\section{Discussion}

The present article discussed three approaches for estimating cross-lagged effects (CL1PM, CL2PM, and RI-CLPM) with a cross-lagged panel design. We applied a causal inference perspective and showed that the traditional CLPM (CL1PM and CL2PM) relies on different assumptions than the RI-CLPM when estimating cross-lagged effects. In the final discussion, we highlight issues that need consideration when choosing between the 


\section{A CRITIQUE OF THE RANDOM INTERCEPT CROSS-LAGGED PANEL MODEL 18}

CLPM and the RI-CLPM in a specific application.

In our discussion of the RI-CLPM, we clarified that, from a causal perspective, there could be two different reasons for using the RI-CLPM to estimate cross-lagged effects. First, one is interested in estimating the causal cross-lagged effect of increasing $X$ by one unit on $Y$ (see Equation (6)) and tries to control for the presence of unobserved confounder variables by including their stable trait factors (see Usami, Murayama, \& Hamaker, 2019). However, we showed in formal analysis and simulated data that the RI-CLPM has only a limited potential to adjust for unobserved confounding. The estimates of the cross-lagged effect will generally be biased unless very restrictive conditions for the effects of the confounder variable are met. Second, one could use the RI-CLPM and change the target of inference by redefining the causal estimand at the within-person level (see Equation (20)). In this case, one would be interested in the causal (within-person) cross-lagged effect of increasing $X$ by a unit around the individual person mean. Even though redefining the causal estimand would, in principle, be possible (Usami, 2020), researchers should be aware that withinperson effects are based on person-mean centered (i.e., ipsatized) scores that only capture temporary fluctuations around individual person means. Thus, they are less appropriate for understanding the potential effects of causes that explain differences between persons. If the main goal is to assess the potential effects of causes that make persons different from other persons - and not from the (long-term) average level - the cross-lagged effect of the CLPM should be preferred (Asendorpf, 2021). However, in our opinion, the within-person effects of the RI-CLPM seem to be better suited for short-term studies of states that typically use shorter time lags (e.g., days) between assessments and are not concerned about systematic long-term changes (see also Orth et al., 2021). Interestingly, causal estimands that are defined at the within-person level seem to be less common in the causal inference literature (see Table 1 in Lundberg, Johnson, \& Stewart, 2021, for a list of common causal estimands). Thus, it would be an important topic for future methodological work to integrate the withinperson perspective of the RI-CLPM into the potential outcome framework of causality (Usami, 2020, 2021; see also Imai \& Kim, 2019).

We confirmed with simulated data the well-known fact (e.g., Finkel, 1995; Little, 2013) that the traditional CLPM is based on a selection-on-observables approach and provides biased estimates if not all relevant covariates are measured (see Scenario C). However, it is less emphasized in the methodological literature that it is often beneficial to include lag-2 effects (i.e., CL2PM) when estimating cross-lagged effects. The main advantage of including lag-2 effects is that allowing for these additional effects of prior measures of $X$ and $Y$ provides a stronger control for the presence of confounding (VanderWeele et al., 2020). However, controlling for prior levels of the exposure $X$ may not always be preferable. If the two assessments of the exposure $X$ are close together in time, the treatment values will show almost no change across time (i.e., very large correlation between $X_{1}$ and $X_{2}$ ). In this case, the prior levels of the exposure (i.e., $X_{1}$ ) would not act as a confounder but as an additional measure of $X_{2}$, resulting in estimates of cross-lagged effects that may be overadjusted by the prior levels of the exposure and difficult to interpret (see VanderWeele et al., 2020, for a discussion of reasons to control for prior measures of $X$ ).

From a more descriptive perspective, it has also been argued that the RI-CLPM provides a more appropriate description of the developmental processes underlying most psychological constructs than the traditional CLPM (Berry \& Willoughby, 2017; Dietvorst 
et al., 2018; Hamaker et al., 2015). This argument builds on ample evidence from longitudinal research using state-trait models (e.g., Geiser, 2021; Cole, 2012) that shows that a stable trait factor explains a substantial proportion of interindividual differences in psychological constructs across time (e.g., Anusic \& Schimmack, 2016; Jansen, Lüdtke, \& Robitzsch, 2020), and is also supported by the fact that the RI-CLPM (that includes stable trait factors for both $X$ and $Y$ ) often provides a better fit than the CL1PM that only includes lag-1 effects (e.g., Bailey et al., 2020; Dietvorst et al., 2018; Oh et al., 2020). We agree that the decomposition of interindividual differences across time into different components of change (e.g., stable traits, time-varying autoregressive traits, and occasion-specific states; see also Kenny \& Zautra, 1995) can provide important insights into the malleability and development of psychological constructs. However, in our view, the goal of modeling development processes should be kept separate from the goal of causal inference. If the goal is causal inference, researchers should be primarily concerned with the ignorability assumption and focus on how the longitudinal data structure can be exploited to provide appropriate control of confounding variables (VanderWeele et al., 2020). If the main interest is in the more descriptive question of modeling development processes, researchers can choose among a plethora of competing statistical approaches that allow modeling different aspects of the dynamics of the change process. For example, it would be possible to include a time factor in the RI-CLPM in addition to the stable trait factors. As pointed out by Usami, Murayama, and Hamaker (2019), this would extend the RI-CLPM to a latent curve model with structured residuals (LCM-SR; Curran, Howard, Bainter, Lane, \& McGinley, 2014) that simultaneously models interindividual differences in linear change across time at the between-person level and cross-lagged effects at the within-person level. It is without question that these hybrid models of change (see Curran \& Hancock, 2021) have great potential to further our understanding of developmental processes. However, in terms of questions of causal inference, it still seems an open question how the appropriate target of causal inference (i.e., causal estimand) for these models should be defined. Furthermore, if one is interested in estimating cross-lagged effects with the LCM-SR, one should be aware that $X$ and $Y$ are not only residualized for the random intercepts (i.e., person means; see Equation (3)) but also for individual differences in linear trends. This further complicates any clear statement of ignorability assumptions (for identifying causal effects) because the cross-lagged effect at the within-person level only captures what is left after accounting for individual differences in growth trends (Usami, Murayama, \& Hamaker, 2019).

We need to mention that our discussion of the cross-lagged panel design has several limitations. First, it should be emphasized that we focused on the causal effect of a variable at a single point in time (i.e., the effect of $X_{2}$ on $Y_{3}$ ). As pointed out before, in the typical cross-lagged panel design, the variable $X$ varies across time, and it would also be possible to estimate the cumulative effect of $X_{1}$ and $X_{2}$ on $Y_{3}$. For example, when assessing the effect of self-esteem on depression, with several waves of data, it would be possible to assess the effects of a treatment trajectory of self-esteem on final depression (i.e., the effect of increasing selfesteem at $\mathrm{T} 1$ and $\mathrm{T} 2$ by one unit on depression at $\mathrm{T} 3$ ). These cumulative effects are rarely investigated in psychological research. One of the few exceptions is VanderWeele, Hawkley, Thisted, and Cacioppo (2011), who investigated the cumulative effects of loneliness on depression using a cross-lagged panel design with five measurement waves (see also Silvey, Demir-Lira, Goldin-Meadow, \& Raudenbush, 2021). However, it should be added that the 
assumptions for identifying the cumulative effect of a treatment trajectory over a series of time points are more demanding than for the effect at a single point in time (Hernán \& Robins, 2020; Robins, Hernán, \& Brumback, 2000; see also Daniel et al., 2013). The main challenge is to adequately control for time-varying confounders (e.g., prior measures of the outcome $Y_{2}$ that may also be affected by prior measures of the treatment $X_{1}$ ). This is an active area of methodological research in longitudinal causal inference (e.g., Keogh, Daniel, VanderWeele, \& Vansteelandt, 2018; Wodtke, 2020).

Second, we focused on a design with only three measurement waves. This allowed us to illustrate the differences between the RI-CLPM and the CLPM for a constellation in which the inclusion of lag-2 effects (CL2PM) resulted in a saturated model. For designs with more than three measurement waves, higher-order lagged effects (e.g., lag-3 effects) could be added so that the CLPM would still be saturated, and it would not be a question of model fit whether the RI-CLPM or the CLPM should be preferred (Reichardt, 2011).

Third, we assumed that the variables are measured without error. In most practical applications, both $X$ and $Y$ will be affected by measurement error, resulting in biased estimates of regression coefficients. Researchers often use multiple indicators (i.e., internal consistency error) to control for measurement error if psychological constructs are measured by multiple items. Multiple indicators can be easily included in the CLPM and RI-CLPM (Little, 2013; Mulder \& Hamaker, 2021). However, internal consistency measures of reliability only focus on measurement error caused by the finite number of items on the test. Both CLPM and RI-CLPM can be extended to account for other sources of error (e.g., short-term fluctuations of the measure or transient error; McCrae, 2015) when estimating cross-lagged effects with error-prone variables (Heise, 1969; Kenny \& Zautra, 1995). However, the main arguments for favoring the CLPM over the RI-CLPM still remain valid in the presence of measurement error.

Finally, we focused in our discussion on the simplified constellation in which all effects between $X$ and $Y$ are linear, and only a single confounder variable exists. In practical applications of the CLPM and RI-CLPM, the selection of covariates and the correct specification of their effects (e.g., interactions and quadratic effects) can be challenging (VanderWeele, 2019). More specifically, one needs the assumption that all relevant covariates are observed. Without specific knowledge about the data-generating process, this assumption is often hard to justify (Imbens \& Rubin, 2015). Therefore, many scholars argued that in real applications, the estimation of causal effects should be accompanied by a sensitivity analysis that tests how sensitive the conclusions are to unmeasured confounding (e.g., Dearing \& Zachrisson, 2019; VanderWeele et al., 2020).

\section{References}

Angrist, J. D., \& Pischke, J.-S. (2009). Mostly harmless econometrics: An empiricist's companion. Princeton University Press. https://doi.org/10.1515/9781400829828

Anusic, I., \& Schimmack, U. (2016). Stability and change of personality traits, selfesteem, and well-being: Introducing the meta-analytic stability and change model of retest correlations. Journal of Personality and Social Psychology, 110(5), 766-781. https://doi.org/10.1037/pspp0000066

Aronow, P. M., \& Miller, B. T. (2019). Foundations of agnostic statistics. Cambridge: Cambridge University Press. https://doi.org/10.1017/9781316831762 
Asendorpf, J. B. (2021). Modeling developmental processes. In J. R. Rauthmann (Ed.), Handbook of personality dynamics and processes (pp. 815-835). London, UK. https://doi.org/10.1016/B978-0-12-813995-0.00031-5

Bailey, D. H., Oh, Y., Farkas, G., Morgan, P., \& Hillemeier, M. (2020). Reciprocal effects of reading and mathematics? Beyond the cross-lagged panel model. Developmental Psychology, 56 (5), 912-921. https://doi.org/10.1037/dev0000902

Berk, R., Brown, L., Buja, A., George, E., Pitkin, E., Zhang, K., \& Zhao, L. (2014). Misspecified mean function regression: Making good use of regression models that are wrong. Sociological Methods \& Research, 43(3), 422-451. https://doi.org/10.1177/0049124114526375

Berry, D., \& Willoughby, M. T. (2017). On the practical interpretability of cross-lagged panel models: Rethinking a developmental workhorse. Child Development, 88(4), 1186-1206. https://doi.org/10.1111/cdev.12660

Bolger, N., \& Laurenceau, J.-P. (2013). Intensive longitudinal methods: An introduction to diary and experience sampling research. New York: Guilford Press.

Cole, D. A. (2012). Latent trait-state models. In R. H. Hoyle (Ed.), Handbook for structural equation modeling (pp. 585-600). New York.

Curran, P. J., \& Hancock, G. R. (2021). The challenge of modeling codevelopmental processes over time. Child Development Perspectives, 15(2), 67-75. https://doi.org/10.1111/cdep.12401

Curran, P. J., Howard, A. L., Bainter, S. A., Lane, S. T., \& McGinley, J. S. (2014). The separation of between-person and within-person components of individual change over time: A latent curve model with structured residuals. Journal of Consulting and Clinical Psychology, 82(5), 879-894. https://doi.org/10.1037/a0035297

Daniel, R. M., Cousens, S. N., De Stavola, B. L., Kenward, M. G., \& Sterne, J. A. C. (2013). Methods for dealing with time-dependent confounding. Statistics in Medicine, 32(9), 1584-1618. https://doi.org/10.1002/sim.5686

Dearing, E., \& Zachrisson, H. D. (2019). Taking selection seriously in correlational studies of child development: A call for sensitivity analyses. Child Development Perspectives, 13(4), 267-273. https://doi.org/10.1111/cdep.12343

Dietvorst, E., Hiemstra, M., Hillegers, M. H. J., \& Keijsers, L. (2018). Adolescent perceptions of parental privacy invasion and adolescent secrecy: An illustration of Simpson's paradox. Child Development, 89(6), 2081-2090. https://doi.org/10.1111/cdev.13002

Ehm, J.-H., Hasselhorn, M., \& Schmiedek, F. (2019). Analyzing the developmental relation of academic self-concept and achievement in elementary school children: Alternative models point to different results. Developmental Psychology, 55(11), 2336-2351. https://doi.org/10.1037/dev0000796

Finkel, S. (1995). Causal analysis with panel data. Thousand Oaks, CA. https://doi.org/10.4135/9781412983594

Geiser, C. (2021). Longitudinal structural equation modeling with Mplus: A latent state-trait perspective. New York: Guilford.

Gische, C., West, S. G., \& Voelkle, M. C. (2021). Forecasting causal effects of interventions versus predicting future outcomes. Structural Equation Modeling, 28(3), 475-492. https://doi.org/10.1080/10705511.2020.1780598

Grimm, K. J., Helm, J., Rodgers, D., \& O'Rourke, H. (2021). Analyzing cross-lag effects: 
A comparison of different cross-lag modeling approaches. New Directions for Child and Adolescent Development, 175, 11-33. https://doi.org/10.1002/cad.20401

Hamaker, E. L., Kuiper, R. M., \& Grasman, R. P. P. P. (2015). A critique of the cross-lagged panel model. Psychological Methods, 20(1), 102-116. https://doi.org/10.1037/a0038889

Heise, D. R. (1969). Separating reliability and stability in test-retest correlation. American Sociological Review, 34, 93-101. https://doi.org/10.2307/2092790

Hernán, M. A., \& Robins, J. M. (2020). Causal inference: What if. Boca Raton: Chapman \& Hall/CRC.

Hirano, K., \& Imbens, G. W. (2004). The propensity score with continuous treatments. In A. Gelman \& X.-L. Meng (Eds.), Applied Bayesian modeling and causal inference from incomplete-data perspectives (pp. 73-84). New York: Wiley. https://doi.org/10.1002/0470090456.ch7

Hoffman, L. (2015). Longitudinal analysis: Modeling within-person fluctuation and change. New York, NY: Routledge/Taylor \& Francis Group.

Imai, K., \& Kim, I. S. (2019). When should we use unit fixed effects regression models for causal inference with longitudinal data? American Journal of Political Science, 63(2), 467-490. https://doi.org/10.1111/ajps.12417

Imbens, G. W. (2004). Nonparametric estimation of average treatment effects under exogeneity: A review. Review of Economics and Statistics, 86(1), 4-29. https://doi.org/10.1162/003465304323023651

Imbens, G. W., \& Rubin, D. B. (2015). Causal inference for statistics, social, and biomedical sciences. New York, NY: Cambridge University Press. https://doi.org/10.1017/CBO9781139025751

Jansen, M., Lüdtke, O., \& Robitzsch, A. (2020). Disentangling different sources of stability and change in students' academic self-concepts: An integrative data analysis using the STARTS model. Journal of Educational Psychology, 112(8), 1614-1631. https://doi.org/10.1037/edu0000448

Kenny, D. A., \& Zautra, A. (1995). The trait-state-error model for multiwave data. Journal of Consulting and Clinical Psychology, 63(1), 52-59. https://doi.org/10.1037/0022006x.63.1.52

Keogh, R. H., Daniel, R. M., VanderWeele, T. J., \& Vansteelandt, S. (2018). Analysis of longitudinal studies with repeated outcome measures: adjusting for time-dependent confounding using conventional methods. American Journal of Epidemiology, 187(5), 1085-1092. https://doi.org/10.1093/aje/kwx311

Kessler, R. C., \& Greenberg, D. F. (1981). Linear panel analysis: Models of quantitative change. New York: Academic Press.

Little, T. D. (2013). Longitudinal structural equation modeling. New York: Guilford Press.

Littlefield, A. K., King, K. M., Acuff, S. F., Foster, K. T., Murphy, J. G., \& Witkiewitz, K. (2021). Limitations of cross-lagged panel models in addiction research and alternative models: An empirical example using project MATCH. Psychology of Addictive Behaviors. ([Epub ahead of print]) https://doi.org/10.1037/adb0000750

Lundberg, I., Johnson, R., \& Stewart, B. M. (2021). What is your estimand? Defining the target quantity connects statistical evidence to theory. American Sociological Review, 86(3), 532-565. https://doi.org/10.1177/00031224211004187 
Marsh, H. W., Pekrun, R., Murayama, K., Arens, A. K., Parker, P. D., Guo, J., \& Dicke, T. (2018). An integrated model of academic self-concept development: Academic selfconcept, grades, test scores, and tracking over 6 years. Developmental Psychology, 54 (2), 263-280. https://doi.org/10.1037/dev0000393

Marsh, H. W., Trautwein, U., Lüdtke, O., Köller, O., \& Baumert, J. (2005). Academic selfconcept, interest, grades, and standardized test scores: Reciprocal effects models of causal ordering. Child Development, 76 (2), 397-416. https://doi.org/10.1111/j.14678624.2005.00853.x

Maxwell, S. E., \& Delaney, H. D. (2004). Designing experiments and analyzing data: A model comparison perspective. Mahwah, NJ: Erlbaum.

McCrae, R. R. (2015). A more nuanced view of reliability: Specificity in the trait hierarchy. Personality and Social Psychology Review, 19(2), 97-112. https://doi.org/10.1177/1088868314541857

Morgan, S. L., \& Winship, C. (2014). Counterfactuals and causal inference: Methods and principles for social research. Cambridge University Press. https://doi.org/10.1017/CBO9781107587991

Mulder, J. D., \& Hamaker, E. L. (2021). Three extensions of the random intercept cross-lagged panel model. Structural Equation Modeling, 28(4), 638-648. https://doi.org/10.1080/10705511.2020.1784738

Mund, M., \& Nestler, S. (2019). Beyond the cross-lagged panel model: Next-generation statistical tools for analyzing interdependencies across the life course. Advances in Life Course Research, 41, 100249. https://doi.org/10.1016/j.alcr.2018.10.002

Newsom, J. T. (2015). Longitudinal structural equation modeling: A comprehensive introduction. New York: Routledge.

Oh, Y., Greenberg, M. T., \& Willoughby, M. T. (2020). Examining longitudinal associations between externalizing and internalizing behavior problems at withinand between-child levels. Journal of Abnormal Child Psychology, 48, 467-480. https://doi.org/10.1007/s10802-019-00614-6

Orth, U., Clark, D. A., Donnellan, M. B., \& Robins, R. W. (2021). Testing prospective effects in longitudinal research: Comparing seven competing crosslagged models. Journal of Personality and Social Psychology, 120(4), 1013-1034. https://doi.org/10.1037/pspp0000358

Pearl, J., Glymour, M., \& Jewell, N. P. (2016). Causal inference in statistics: A primer. John Wiley \& Sons.

Plewis, I. (1985). Analysing change: Methods for the measurement and explanation of change in the social sciences. Chichester, UK: Wiley.

Reichardt, C. S. (2011). Commentary: Are three waves of data sufficient for assessing mediation? Multivariate Behavioral Research, 46(5), 842-851. https://doi.org/10.1080/00273171.2011.606740

Reichardt, C. S. (2019). Quasi-experimentation: A guide to design and analysis. New York, NY: Guilford Press.

Robins, J. M., Hernán, M. A., \& Brumback, B. (2000). Marginal structural models and causal inference in epidemiology. Epidemiology, 11, 550-560. https://doi.org/10.1097/00001648-200009000-00011

Rosseel, Y. (2012). lavaan: An R package for structural equation modeling. Journal of 
Statistical Software, 48(2), 1-36. https://doi.org/10.18637/jss.v048.i02

Ruzek, E. A., \& Schenke, K. (2019). The tenuous link between classroom perceptions and motivation: A within-person longitudinal study. Journal of Educational Psychology, 111(5), 903-917. https://doi.org/10.1037/edu0000323

Silvey, C., Demir-Lira, Ö. E., Goldin-Meadow, S., \& Raudenbush, S. W. (2021). Effects of time-varying parent input on children's language outcomes differ for vocabulary and syntax. Psychological Science, 32(4), 536-548.

Usami, S. (2020). Within-person variability score-based causal inference: A two-step semiparametric estimation for joint effects of time-varying treatments. arXiv Preprint, arxXiv:2007.03973v1.

Usami, S. (2021). On the differences between general cross-lagged panel model and random-intercept cross-lagged panel model: Interpretation of cross-lagged parameters and model choice. Structural Equation Modeling, 28(3), 331-344. https://doi.org/10.1080/10705511.2020.1821690

Usami, S., Murayama, K., \& Hamaker, E. L. (2019). A unified framework of longitudinal models to examine reciprocal relations. Psychological Methods, 24(5), 637-657. https://doi.org/10.1037/met0000210

Usami, S., Todo, N., \& Murayama, K. (2019). Modeling reciprocal effects in medical research: Critical discussion on the current practices and potential alternative models. PloS ONE, 14(9), e0209133. https://doi.org/10.1371/journal.pone.0209133

VanderWeele, T. J. (2015). Explanation in causal inference: Methods for mediation and interaction. Oxford: Oxford University Press.

VanderWeele, T. J. (2019). Principles of confounder selection. European Journal of Epidemiology, 34 (3), 211-219. https://doi.org/10.1007/s10654-019-00494-6

VanderWeele, T. J., Hawkley, L. C., Thisted, R. A., \& Cacioppo, J. T. (2011). A marginal structural model analysis for loneliness: Implications for intervention trials and clinical practice. Journal of Consulting and Clinical Psychology, 79(2), 225-235. https://doi.org/10.1037/a0022610

VanderWeele, T. J., Jackson, J. W., \& Li, S. (2016). Causal inference and longitudinal data: A case study of religion and mental health. Social Psychiatry and Psychiatric Epidemiology, 51(11), 1457-1466. https://doi.org/10.1007/s00127-016-1281-9

VanderWeele, T. J., Mathur, M. B., \& Chen, Y. (2020). Outcome-wide longitudinal designs for causal inference: A new template for empirical studies. Statistical Science, 35(3), 437-466. https://doi.org/10.1214/19-STS728

Vegetabile, B. G., Griffin, B. A., Coffman, D. L., Cefalu, M., Robbins, M. W., \& McCaffrey, D. F. (2021). Nonparametric estimation of population average dose-response curves using entropy balancing weights for continuous exposures. Health Services and Outcomes Research Methodology, 21(1), 69-110. https://doi.org/10.1007/s10742020-00236-2

West, S. G., Taylor, A. B., \& Wu, W. (2012). Model fit and model selection in structural equation modeling. In R. H. Hoyle (Ed.), Handbook of structural equation modeling (pp. 209-231). Guilford Press.

Wodtke, G. T. (2020). Regression-based adjustment for time-varying confounders. Sociological Methods 83 Research, 49(4), 906-946. https://doi.org/10.1177/004912411876908

Wooldridge, J. M. (2010). Econometric analysis of cross section and panel data. Cambridge, 
MA: MIT Press.

Zhou, N., Cao, H., Liu, F., Wu, L., Liang, Y., Xu, J., ... Zhang, J. (2020). A four-wave, cross-lagged model of problematic internet use and mental health among Chinese college students: Disaggregation of within-person and between-person effects. Developmental Psychology, 56(5), 1009-1021. https://doi.org/10.1037/dev0000907

Zyphur, M. J., Allison, P. D., Tay, L., Voelkle, M. C., Preacher, K. J., Zhang, Z., ... Diener, E. (2020). From data to causes I: Building a general crosslagged panel model (GCLM). Organizational Research Methods, 23(4), 651-687. https://doi.org/10.1177/1094428119847278

\section{Appendix: Formal Analysis of Causal Implications of the RI-CLPM}

We start with writing the RI-CLPM for the outcome $Y_{i 3}$ (see Equation (16)):

$$
Y_{i 3}=U_{Y i}+\beta_{Y_{3}^{*} Y_{2}^{*}} Y_{i 2}^{*}+\beta_{Y_{3}^{*} X_{2}^{*}} X_{i 2}^{*}+\delta_{Y_{3} i}
$$

It should be emphasized that $X_{i 2}^{*}$ and $Y_{i 2}^{*}$ are the decomposed within-person variables (see

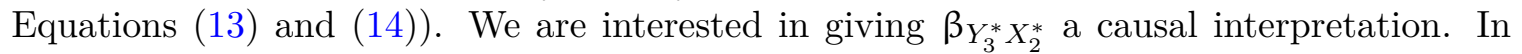
their formal analysis of the causal implications of the RI-CLPM, Usami, Murayama, and Hamaker (2019) stated the following extended ignorability assumption (see Assumption 2' on p. 651):

$$
Y_{i 3}\left(x_{2}\right) \perp X_{i 2} \mid Y_{i 2}, U_{Y i}
$$

Note that the extended ignorability assumption is stated for the undecomposed variables $X_{i 2}$ and $Y_{i 2}$. They also made the simplifying assumption of linear effects so that given the assumption in Equation (A2) the expected potential outcomes for $Y_{i 3}$ can be expressed as (see Equation 24 in Usami, Murayama, \& Hamaker, 2019):

$$
\begin{aligned}
\mathrm{E}\left(Y_{i 3}\left(x_{2}\right)\right) & =U_{Y i}+\gamma_{Y_{3} Y_{2}} Y_{i 2}+\gamma_{Y_{3} X_{2}} X_{i 2} \\
& =\left(1+\gamma_{Y_{3} Y_{2}}\right) U_{Y i}+\gamma_{Y_{3} X_{2}} U_{X i}+\gamma_{Y_{3} Y_{2}} Y_{i 2}^{*}+\gamma_{Y_{3} X_{2}} X_{i 2}^{*}
\end{aligned}
$$

Comparing the coefficients between Equation (A1) and Equation (A3) reveals that $\beta_{Y_{3}^{*} X_{2}^{*}}$ is an unbiased estimate of $\gamma_{Y_{3} X_{2}}$ if the following condition is met:

$$
U_{Y i}=\left(1+\gamma_{Y_{3} Y_{2}}\right) U_{Y i}+\gamma_{Y_{3} X_{2}} U_{X i}
$$

This can be rewritten as $\gamma_{Y_{3} Y_{2}} U_{Y i}+\gamma_{Y_{3} X_{2}} U_{X i}=0$, or

$$
U_{Y i}=-\frac{\gamma_{Y_{3} X_{2}}}{\gamma_{Y_{3} Y_{2}}} U_{X i}
$$

Thus, the stable traits need to be perfectly negatively correlated, that is, $\operatorname{Cor}\left(U_{Y i}, U_{X i}\right)=-1$, with $\gamma_{Y_{3} Y_{2}}^{2} \operatorname{Var}\left(U_{Y i}\right)=\gamma_{Y_{3} X_{2}}^{2} \operatorname{Var}\left(U_{X i}\right)$. This condition seems to be unlikely to hold in empirical applications. 